PROCEEDINGS OF THE

AMERICAN MATHEMATICAL SOCIETY

Volume 141, Number 6, June 2013, Pages 1849-1861

S 0002-9939(2012)11449-2

Article electronically published on December 4, 2012

\title{
CONTRACTORS FOR FLOWS
}

\author{
DELIA GARIJO, ANDREW GOODALL, AND JAROSLAV NEŠETŘIL
}

(Communicated by Jim Haglund)

\begin{abstract}
We answer a question raised by Lovász and B. Szegedy [Contractors and connectors in graph algebras, J. Graph Theory 60:1 (2009)] asking for a contractor for the graph parameter counting the number of $B$-flows of a graph, where $B$ is a subset of a finite Abelian group closed under inverses. We prove our main result using the duality between flows and tensions and finite Fourier analysis.
\end{abstract}

\section{INTRODUCTION}

In their paper 11] Lovász and B. Szegedy introduce the notion of connectors and contractors in quantum graph algebras. Connectors and contractors for a graph parameter allow expressions for the parameter of a graph obtained by the connection of two vertices by an edge or the contraction of an edge (either operation possibly producing multiple edges or loops, respectively) in terms of values of the parameter on graphs obtained by operations that preserve simplicity. For example, a connector for the flow polynomial is any path of length two or more. Contractors for graph parameters can be thought of as a generalization of the deletion-contraction identity for the chromatic polynomial, flow polynomial, and other specializations of the Tutte polynomial.

In [11, Section 2.3] Lovász and B. Szegedy comment that "there does not seem to be a simple explicit construction for a contractor" for the number of $B$-flows, where $B$ is a subset of a finite Abelian group closed under inverses. In Section 4 we give an explicit construction of such contractors.

We prove our result using the Fourier transform on finite Abelian groups. Towards this end, in Section 2 we introduce homomorphism functions, flows and tensions of a graph, contractors in graph algebras, and then describe those properties of the Fourier transform that will be used to enable us to reach the main result (Theorem 3.10 and its special case for $B$-flows, Theorem 4.1). Using the Fourier transform we can express any weighted function on the set of tensions of a graph (effectively a homomorphism function to an edge-weighted graph) as a related weighted function on the set of flows of the graph. This in particular allows the number of $B$-flows to be written as the number of homomorphisms to an edgeweighted graph (Lemma 2.3). Although the duality relationship between flows and

Received by the editors January 13, 2011 and, in revised form, September 15, 2011.

2010 Mathematics Subject Classification. Primary 05C21, 05C25; Secondary 05C99.

Key words and phrases. Graph homomorphism, Fourier transform, contractor, flows, tensions.

The first author's research supported by projects O.R.I MTM2008-05866-C03-01 and PAI FQM-0164.

The second and third authors' research supported by ITI 1M0545 and the Centre for Discrete Mathematics, Theoretical Computer Science and Applications (DIMATIA).

(C) 2012 American Mathematical Society 1849

Reverts to public domain 28 years from publication 
tensions is well known, there have not been many useful applications of this correspondence. In this paper it allows us to transform a special type of instance of a problem we do not in general know yet how to solve (Problem 5.1 in Section 5) into one that we can solve.

For graphs $G$ and $H$, let hom $(G, H)$ denote the number of homomorphisms from $G$ to $H$. The definition can be extended to edge-weighted graphs $H$, as explained in Section 2.1. In Section 3 we consider the problem of finding a contractor for the homomorphism function $\operatorname{hom}(\cdot, H)$. The minimal polynomial of the adjacency matrix of $H$ can be used to construct a contractor for $\operatorname{hom}(\cdot, H)$ if the adjacency matrix of $H$ does not have eigenvalue 0 (Theorem 3.3). Theorem 3.4 gives a contractor for $\operatorname{hom}(\cdot, H)$ when the adjacency matrix of $H$ has no diagonal entry equal to an off-diagonal entry. The relevant edge-weighted graph $H$ that gives the number of $B$-flows turns out to have eigenvalue 0 , and when $B$ is a strict subset of nonzero group values there are off-diagonal zero entries as well as zeroes on the diagonal of the adjacency matrix of $H$. However, the special structure of $H$ as an edge-weighted Cayley graph allows us to obtain a contractor by using the minimal polynomial of the graph whose edge weights are obtained by taking the Fourier transform of the edge weights of $H$ (Theorem 3.10). In Section 4 we apply the theory developed in Sections 2 and 3 to construct a contractor for the number of $B$-flows.

In Section 5 we conclude by outlining some possible directions for future research. A sequel to this paper, which includes the application of our results to Jaeger's $B$ flow problems [9], is in preparation [5].

\section{Preliminaries}

2.1. Homomorphism functions. Given two graphs $G=(V(G), E(G))$ and $H=$ $(V(H), E(H))$, a homomorphism from $G$ to $H$, written as $\psi: G \rightarrow H$, is a mapping $\psi: V(G) \rightarrow V(H)$ such that $\psi(u) \psi(v) \in E(H)$ whenever $u v \in E(G)$. The graph parameter $\operatorname{hom}(G, H)$ counting the number of homomorphisms from $G$ to $H$ can be generalized to edge-weighted graphs $H$ as follows. (We do not need to consider the more general case where $H$ has vertex weights as well as edge weights, as is done in [11, since the relevant graphs $H$ for counting $B$-flows do not require them.)

Let $H$ be a graph with a real weight $\beta_{H}(i j)$ associated with each edge $i j \in E(H)$ and let $G$ be an unweighted graph.

The homomorphism function $\operatorname{hom}(G, H)$ is defined by

$$
\operatorname{hom}(G, H)=\sum_{\psi: V(G) \rightarrow V(H)} \prod_{u v \in E(G)} \beta_{H}(\psi(u), \psi(v)) .
$$

If we fix $U \subseteq V(G)$ and define for every function $\phi: U \rightarrow V(H)$ the weight

$$
\operatorname{hom}_{\phi}(G, H)=\sum_{\substack{\psi: V(G) \rightarrow V(H) \\ \psi_{\mid U}=\phi}} \prod_{u v \in E(G)} \beta_{H}(\psi(u), \psi(v)),
$$

then it is easy to see that we have the decomposition

$$
\operatorname{hom}(G, H)=\sum_{\phi: U \rightarrow V(H)} \operatorname{hom}_{\phi}(G, H) .
$$


2.2. Flows and tensions. Let $G=(V, E)$ be a graph with an arbitrary fixed orientation of its edges. Denote by $c(G)$ the number of connected components of $G$, and by $r(G)=|V|-c(G)$ its rank. Let $D$ be the $V \times E$ incidence matrix of $G$, with $(v, e)$-entry given by

$$
D_{v, e}= \begin{cases}+1 & v \text { is the head of } e \\ -1 & v \text { is the tail of } e \\ 0 \quad & v \text { is not an endpoint of } e, \text { or } e \text { is a loop on } v\end{cases}
$$

Suppose $\Gamma$ is a finite commutative ring with unity, and let $\Gamma^{V}=\{f: V \rightarrow \Gamma\}$ and $\Gamma^{E}=\{g: E \rightarrow \Gamma\}$.

The linear transformation $D: \Gamma^{E} \rightarrow \Gamma^{V}$ of $\Gamma$-modules has kernel equal to the set of $\Gamma$-flows of $G$. This is to say that a function $g: E \rightarrow \Gamma$ is a $\Gamma$-flow if and only if, for each vertex $v \in V$,

$$
\sum_{\substack{e=u v \\ v \text { head of } e}} g(e)-\sum_{\substack{e=u v \\ v \text { tail of } e}} g(e)=0 .
$$

If $B=-B$ is a subset of $\Gamma$, then a $B$-flow of $G$ is a $\Gamma$-flow taking values in $B$. (We have followed Jaeger's [9] usage of the term $B$-flow; in [1] Lovász and B. Szegedy use the letter $S$ instead of $B$, and so speak of $S$-flows rather than $B$ flows.) In particular, when $B=\Gamma \backslash\{0\}$, a $B$-flow is a nowhere-zero $\Gamma$-flow of $G$. It is well known that the number of nowhere-zero $\Gamma$-flows of $G$ depends only on $|\Gamma|$ and is given by the evaluation of the flow polynomial $F(G ; z)$ at $z=|\Gamma|$.

The transpose linear transformation $D^{\top}: \Gamma^{V} \rightarrow \Gamma^{E}$ has as image the set of $\Gamma$-tensions of $G$. A $\Gamma$-tension $g: E \rightarrow \Gamma$ arises from a vertex $\Gamma$-colouring $f: V \rightarrow \Gamma$ of $G$, by setting $g(e)=f(v)-f(u)$ for each edge $e$ with tail $u$ and head $v$.

A $B$-tension of $G$ is a $\Gamma$-tension of $G$ taking values in $B$. In particular, when $B=\Gamma \backslash\{0\}$, a $B$-tension arises from $|\Gamma|^{c(G)}$ proper vertex $\Gamma$-colourings of $G$. The number of proper vertex $\Gamma$-colourings is given by the evaluation of the chromatic polynomial $P(G ; z)$ at $z=|\Gamma|$.

To define $\Gamma$-flows and $\Gamma$-tensions all that is needed is the structure of $\Gamma$ as an additive Abelian group. The set of $\Gamma$-flows forms an additive subgroup of $\Gamma^{E}$ isomorphic to $\Gamma^{|E|-r(G)}$ and the set of $\Gamma$-tensions a subgroup of $\Gamma^{E}$ isomorphic to $\Gamma^{r(G)}$. As we shall see in Section 2.4 the extra ring structure on $\Gamma$ allows us to relate the sets of tensions and flows to each other as orthogonal complements.

2.3. Contractors. For a fixed positive integer $k$, a $k$-labelled graph $(G, \lambda)$ is a finite graph $G$ (without loops but possibly multiple edges) together with a function $\lambda:[k] \rightarrow V(G)$. Lovász and Szegedy [1] restrict $\lambda$ to be an injective function, but we shall follow Schrijver [12] and allow vertices to have multiple labels. A $k$-labelled graph $(G, \lambda)$ is simple if $G$ has no parallel edges, the function $\lambda$ is injective and its image is an independent set of vertices.

A $k$-labelled quantum graph is a formal linear combination of $k$-labelled graphs with coefficients in $\mathbb{R}$. Let $\mathcal{G}_{k}$ denote the set of $k$-labelled quantum graphs, $\mathcal{G}_{k}^{0}$ the subset of $k$-labelled quantum graphs whose labelled vertices are independent, and $\mathcal{G}_{k}^{\text {simp }}$ the subset of simple $k$-labelled quantum graphs.

We let $K_{n}$ denote the complete graph on $n$ vertices and $\bar{K}_{n}$ its complement, consisting of $n$ isolated vertices. 
For two $k$-labelled graphs $X$ and $Y$, the product $X Y$ is defined [11 by taking the disjoint union of $X$ and $Y$ and then identifying vertices which share the same label. The product is associative and commutative and extends linearly to $\mathcal{G}_{k}$. The identity for this multiplication on $\mathcal{G}_{k}$ is $\left(\bar{K}_{k}, \lambda\right)$, where $\lambda$ is injective, i.e., $k$ independent vertices each with one label.

In the sequel we shall just consider the case of 2-labelled graphs, i.e., $k=2$.

A further binary operation on 2-labelled graphs is the concatenation $X \circ Y$ of $X$ and $Y$, defined by identifying the vertex of $X$ labelled 2 with the vertex labelled 1 in $Y$ and unlabelling this merged vertex. Concatentation is associative but not commutative. It is extended linearly to $\mathcal{G}_{2}$. The identity for this multiplication on $\mathcal{G}_{2}$ is $K_{1}$, with both labels 1 and 2 on the single vertex.

Let $P_{\ell}, \ell \geq 1$, denote the 2-labelled path on $\ell+1$ vertices with one endpoint labelled 1 and the other endpoint labelled 2. In this notation, $P_{1}=K_{2}$. We have $P_{\ell} \circ P_{m}=P_{\ell+m}$, and $P_{\ell} P_{m}=C_{\ell+m}$, where the cycle has labels spaced $\ell$ edges apart in one direction, $m$ edges apart in the other. If $X$ is a 2-labelled graph, then $X P_{1}$ is the 2-labelled graph with an edge added joining the vertices labelled 1 and 2 . In particular, if $X$ is a simple 2-labelled graph, then $X P_{1}$ joins nonadjacent vertices with an edge.

The graph $K_{1}$ with vertex carrying both labels 1 and 2 when applied as a product with $X$ (giving the graph $X K_{1}$ ) identifies the vertices labelled 1 and 2 in $X$ (or does nothing if both labels are on a single vertex in $X$ ). The graph $X K_{1}$ is equal to the graph $X P_{1}$ with the edge that joins 1 and 2 (the edge not belonging to $X$ ) contracted. If vertices 1 and 2 are adjacent in $X$, then $X P_{1}$ has parallel edges joining 1 and 2 , and $X K_{1}$ has a loop for each edge between 1 and 2 in $X$.

The graph $\bar{K}_{2}$ with one vertex labelled 1 and the other labelled 2 is the identity for the product of 2-labelled graphs.

A series-parallel 2-labelled graph is obtained from $P_{1}$ by a sequence of products (parallel extensions) and concatenations (series extensions).

Definition 2.1 ([1]). Let $h$ be a graph parameter. A 2-labelled quantum graph $Z \in \mathcal{G}_{2}$ is a contractor for $h$ if for all $X \in \mathcal{G}_{2}^{0}$ we have

$$
h(X Z)=h\left(X K_{1}\right)
$$

Informally, attaching a contractor $Z$ at two nonadjacent vertices acts like identifying these vertices as far as the value of the graph parameter $h$ is concerned.

Example 2.2. The deletion-contraction relation for the chromatic polynomial takes the form $P\left(X P_{1} ; z\right)=P(X ; z)-P\left(X K_{1} ; z\right)$, where $X$ is a 2-labelled graph. When the labelled vertices are adjacent in $X$ so that $X K_{1}$ has a loop, this relation reduces to $P\left(X P_{1} ; z\right)=P(X ; z)$. Extending the chromatic polynomial linearly to $\mathcal{G}_{2}$, we then have $P\left(X K_{1} ; z\right)=P\left(X\left(\bar{K}_{2}-P_{1}\right) ; z\right)$ for $X \in \mathcal{G}_{2}$, and in particular for $X \in \mathcal{G}_{2}^{0}$. Thus, the quantum graph $\bar{K}_{2}-P_{1}$ is a contractor for the chromatic polynomial.

2.4. B-flows by homomorphisms using Fourier analysis. In this section we develop the connection between flows and tensions in the context of Fourier analysis. Our main theorem in this section is Lemma 2.3, which expresses the number of $B$ flows in terms of homomorphisms to a weighted Cayley graph. In order to reach 
this result we have to briefly introduce the relevant concepts from finite Fourier analysis, for more on which see e.g. [13. The duality between flows and tensions is well known, especially in statistical physics (see e.g. 2]), with an early example being Van der Waerden's Eulerian subgraph expansion of the Ising model partition function [14].

Let $\Gamma \cong \mathbb{Z}_{n_{1}} \oplus \mathbb{Z}_{n_{2}} \oplus \cdots \oplus \mathbb{Z}_{n_{r}}$ be a finite additive Abelian group of order $n=n_{1} n_{2} \cdots n_{r}$. A character of $\Gamma$ is a (group) homomorphism $\chi: \Gamma \rightarrow \mathbb{C}^{\times}$, where $\mathbb{C}^{\times}$is the multiplicative group of the field of complex numbers. The set of characters of $\Gamma$ forms a group $\widehat{\Gamma}$ under pointwise multiplication which is isomorphic to $\Gamma$. For each $x \in \Gamma$, let $\chi_{x}$ denote the image of $x$ under a fixed isomorphism $\Gamma \rightarrow \widehat{\Gamma}$. In particular, the trivial character $\chi_{0}$ is defined by $\chi_{0}(y)=1$ for all $y \in \Gamma$, and $\chi_{-x}(y)=\overline{\chi_{x}(y)}$, the bar denoting complex conjugation.

Denote by $\mathbb{C}^{\Gamma}$ the vector space over $\mathbb{C}$ of all functions from $\Gamma$ to $\mathbb{C}$ which has Hermitian inner product defined for $\alpha, \beta \in \mathbb{C}^{\Gamma}$ by

$$
\langle\alpha, \beta\rangle=\sum_{x \in \Gamma} \alpha(x) \overline{\beta(x)} .
$$

The vector space $\mathbb{C}^{\Gamma}$ has the additional structure of an algebra under either of the following two definitions of multiplication:

(i) the pointwise product $\alpha \cdot \beta$ of $\alpha, \beta \in \mathbb{C}^{\Gamma}$, defined for $x \in \Gamma$ by $\alpha \cdot \beta(x)=$ $\alpha(x) \beta(x)$,

(ii) the convolution $\alpha * \beta$ of $\alpha, \beta \in \mathbb{C}^{\Gamma}$, defined for $x \in \Gamma$ by

$$
\alpha * \beta(x)=\sum_{y \in \Gamma} \alpha(y) \beta(x-y) .
$$

The set $\left\{\delta_{x}: x \in \Gamma\right\}$ of indicator functions defined by

$$
\delta_{x}(y)= \begin{cases}1 & x=y \\ 0 & x \neq y\end{cases}
$$

forms an orthonormal basis for $\mathbb{C}^{\Gamma}$, with $\left\langle\delta_{x}, \delta_{y}\right\rangle=\delta_{x}(y)$. We extend the indicator function notation to subsets $B$ of $\Gamma$, defining $\delta_{B}=\sum_{x \in B} \delta_{x}$.

The characters of $\Gamma$ are also orthogonal in this inner product space, with $\left\langle\chi_{x}, \chi_{y}\right\rangle$ $=|\Gamma| \delta_{x}(y)$.

For $\alpha \in \mathbb{C}^{\Gamma}$, the Fourier transform $\widehat{\alpha} \in \mathbb{C}^{\Gamma}$ is defined for $y \in \Gamma$ by

$$
\widehat{\alpha}(y)=\left\langle\alpha, \chi_{y}\right\rangle=\sum_{x \in \Gamma} \alpha(x) \chi_{y}(-x) .
$$

The Fourier transform maps the basis of indicator functions to the basis of characters: $\widehat{\delta_{y}}=\chi_{-y}$. The Fourier inversion formula $\widehat{\widehat{\alpha}}(x)=|\Gamma| \alpha(-x)$ gives the inverse transform

$$
\alpha(x)=\frac{1}{|\Gamma|}\left\langle\widehat{\alpha}, \chi_{-x}\right\rangle=\frac{1}{|\Gamma|} \sum_{y \in \Gamma} \widehat{\alpha}(y) \chi_{x}(y)
$$

Note that $\widehat{\delta_{\Gamma}}=|\Gamma| \delta_{0}$ and $\widehat{\delta_{0}}=\delta_{\Gamma}$, since $\left\langle\delta_{\Gamma}, \chi_{y}\right\rangle=\left\langle\chi_{0}, \chi_{y}\right\rangle=|\Gamma| \delta_{0}(y)$. 
The Fourier transform gives an isomorphism of the algebra $\mathbb{C}^{\Gamma}$ with multiplication pointwise product with the algebra $\mathbb{C}^{\Gamma}$ with multiplication convolution: for $y \in \Gamma$,

$$
\begin{gathered}
\widehat{\alpha * \beta}(y)=\widehat{\alpha} \cdot \widehat{\beta}(y), \\
\widehat{\alpha \cdot \beta}(y)=\frac{1}{|\Gamma|} \widehat{\alpha} * \widehat{\beta}(y) .
\end{gathered}
$$

We now give $\Gamma$ the further structure of a ring by defining multiplication of elements $x=\left(x_{1}, x_{2}, \ldots, x_{r}\right)$ and $y=\left(y_{1}, y_{2}, \ldots, y_{r}\right)$ of $\Gamma$ componentwise, setting $x y=\left(x_{1} y_{1}, x_{2} y_{2}, \ldots, x_{r} y_{r}\right)$.

A character $\chi \in \widehat{\Gamma}$ is a generating character for $\Gamma$ if $\chi_{x}(y)=\chi(x y)$ for each character $\chi_{x} \in \widehat{\Gamma}$. A generating character of the ring $\mathbb{Z}_{n}$ is defined by $\chi(z)=\omega^{z}$, where $\omega$ is any primitive $n$th root of unity. If $\chi_{1}$ is a generating character for $\Gamma_{1}$ and $\chi_{2}$ a generating character for $\Gamma_{2}$, then $\chi=\chi_{1} \oplus \chi_{2}$ defined by $\chi\left(x_{1}, x_{2}\right)=$ $\chi_{1}\left(x_{1}\right) \chi_{2}\left(x_{2}\right)$ is a generating character for $\Gamma_{1} \oplus \Gamma_{2}$.

Consider $\Gamma^{m}$, the $m$-fold direct sum of $\Gamma$, and suppose $\psi$ is a generating character for $\Gamma$. Then $\chi$ defined by $\chi\left(x_{1}, \ldots, x_{m}\right)=\psi\left(x_{1}\right) \cdots \psi\left(x_{m}\right)$ for $\left(x_{1}, \ldots, x_{m}\right) \in \Gamma^{m}$ is a generating character for $\Gamma^{m}$. The Euclidean inner product (dot product) is defined for $x=\left(x_{1}, \ldots, x_{m}\right), y=\left(y_{1}, \ldots, y_{m}\right) \in \Gamma^{m}$ by $x \cdot y=x_{1} y_{1}+\cdots+x_{m} y_{m}$. Since $\psi\left(x_{1}\right) \cdots \psi\left(x_{m}\right)=\psi\left(x_{1}+\cdots+x_{m}\right)$, it follows that $\chi_{x}(y)=\chi(x y)=\psi(x \cdot y)$ for $x, y \in \Gamma^{m}$. The orthogonal to a subset $C$ of $\Gamma^{m}$ (with respect to the Euclidean inner product) is defined by $C^{\perp}=\{y \in \Gamma: x \cdot y=0$ for all $y \in C\}$.

For a subgroup $C$ of $\Gamma$, the annihilator of $C$ is defined by $C^{\sharp}=\left\{x \in R: \chi_{x}(y)=\right.$ 1 for all $y \in C\}$. The annihilator $C^{\sharp}$ is a subgroup of $\Gamma$ isomorphic to $\Gamma / C$. When considering a subgroup $C$ of $\Gamma^{m}$, we have $C^{\perp} \subseteq C^{\sharp}$, since $\chi_{x}(y)=\psi(x \cdot y)=$ $\psi(0)=1$ for all $y \in C$ whenever $x \in C^{\perp}$, where $\psi$ is a suitably defined generating character of $\Gamma$. For our choice of multiplicative structure on $\Gamma$ the reverse inclusion holds too, although we do not need to prove this fact here. (The equality $C^{\perp}=C^{\sharp}$ holds generally for $C \subseteq \Gamma^{m}$ when $\Gamma$ is a Frobenius ring; see for example Section 7 of 15.$)$

A key property of the Fourier transform is that it takes indicators of subgroups to (scalar multiples of) indicators of their annihilators:

$$
\widehat{\delta}_{C}(y)=\sum_{x \in C} \chi_{x}(y)=|C| \delta_{C^{\sharp}}(y) .
$$

Following from (2.2), (2.3) and (2.5) is the Poisson summation formula: for every $\alpha \in \mathbb{C}^{\Gamma}$,

$$
\sum_{x \in C} \alpha(x+z)=\frac{1}{\left|C^{\sharp}\right|} \sum_{x \in C^{\sharp}} \widehat{\alpha}(x) \chi_{z}(x) .
$$

We now apply the machinery we have set up to relate flows and tensions of a graph.

Let $D$ be the incidence matrix of a graph $G$, as defined in Section 2.2 . Recall that the kernel $\operatorname{ker} D$ of the map $D: \Gamma^{E} \rightarrow \Gamma^{V}$ consists of the $\Gamma$-flows of $G$, forming an additive group isomorphic to $\Gamma^{|E|-r(G)}$. Likewise, the image $\operatorname{im} D^{\top}$ of the adjoint map $D^{\top}: \Gamma^{V} \rightarrow \Gamma^{E}$ consists of the $\Gamma$-tensions of $G$, isomorphic as an additive group to $\Gamma^{r(G)}$. 
Put $C=\operatorname{ker} D \subseteq \Gamma^{E}$. We have $C^{\sharp} \supseteq C^{\perp} \supseteq \operatorname{im} D^{\top}$, the latter inclusion since $x \cdot D^{\top} y=D x \cdot y$, so that whenever $x \in \operatorname{ker} D$ we have $x \cdot D^{\top} y=0$. With $\left|\operatorname{im} D^{\top}\right|=|\Gamma|^{r(G)}=\left|\Gamma^{E} / C\right|=\left|C^{\sharp}\right|$, we have $\left|C^{\sharp}\right|=\left|C^{\perp}\right|$. Hence $C^{\sharp}=C^{\perp}$.

From equation (2.6) it follows that, for any $\alpha \in \mathbb{C}^{\Gamma}$,

$$
\sum_{x \in \operatorname{ker} D} \alpha(x)=\frac{1}{\mid \Gamma^{r(G)}} \sum_{y \in \operatorname{im} D^{\top}} \widehat{\alpha}(y) .
$$

Lemma 2.3. Let $B=-B$ be a subset of an additive Abelian group $\Gamma$. Let $H=$ Cayley $(\Gamma, B)$ be the graph on vertex set $\Gamma$ with an edge joining vertices $i$ and $j$ if and only if $j-i \in B$, and let $\widehat{H}$ be the edge-weighted Cayley graph on vertex set $\Gamma$ with edge ij having weight $\widehat{\delta}_{B}(j-i)$.

Then the number of B-tensions of a graph $G=(V, E)$ is $|\Gamma|^{-c(G)} \operatorname{hom}(G, H)$, and the number of $B$-flows of $G$ is $|\Gamma|^{-|V|} \operatorname{hom}(G, \widehat{H})$.

Proof. A function $f: V \rightarrow \Gamma$ is a homomorphism contributing to $\operatorname{hom}(G, H)$ if and only if $f(v)-f(u) \in B$ for each edge $e=u v \in E$, which is to say that $g(e):=f(v)-f(u)$ is a $B$-tension of $G$. For a given $B$-tension $g: E \rightarrow \Gamma$ there are $|\Gamma|^{c(G)}$ vertex colourings $f: V \rightarrow \Gamma$ with $D f=g$. This proves that the number of $B$-tensions is $|\Gamma|^{-c(G)} \operatorname{hom}(G, H)$.

To prove that $|\Gamma|^{-|V|} \operatorname{hom}(G, \widehat{H})$ is the number of $B$-flows, take $\alpha=\delta_{B^{E}}$ in (2.7), which has Fourier transform for $g: E \rightarrow \Gamma$ given by

$$
\widehat{\delta}_{B^{E}}(g)=\prod_{e \in E} \widehat{\delta}_{B}(g(e)),
$$

which yields

$$
\left|\operatorname{ker} D \cap B^{E}\right|=\frac{1}{|\Gamma|^{|V|}} \sum_{f: V \rightarrow \Gamma} \prod_{u v \in E} \widehat{\delta_{B}}(f(v)-f(u)) \text {. }
$$

\section{Contractors For homomorphism FUnCtions}

After the preliminaries of Section 2 we now turn to the general problem of finding a contractor for hom $(\cdot, H)$. (In Section 4 we then consider the special case of finding a contractor for the number of $B$-flows.)

Let $H$ be an edge-weighted graph with adjacency matrix $A=(\beta(i j))$. To every $X \in \mathcal{G}_{2}$ assign a $V(H) \times V(H)$-matrix $M(X)=M_{H}(X)$ with $(i, j)$-entry equal to $\operatorname{hom}_{\phi}(X, H)$, where $\phi(1)=i$ and $\phi(2)=j$. As $H$ is fixed we shall usually leave the dependence on $H$ implicit and write $M(X)$ instead of $M_{H}(X)$. It is easy to see that the function $M: \mathcal{G}_{2} \rightarrow \mathbb{C}^{V(H) \times V(H)}$ is linear and satisfies

$$
M(X \circ Y)=M(X) M(Y), M(X Y)=M(X) \circ M(Y),
$$

where (for matrices) $\circ$ is the entrywise (Hadamard, or Schur) product.

Let $P_{k}$ denote the path on $k$ edges with endpoints labelled 1 and 2 . Observe that $M\left(P_{1}\right)=(\beta(i j))$ is the adjacency matrix of $H$.

The graph $P_{1}^{k}$ ( $k$-fold product of $P_{1}$ with itself $)$ is a thick edge, consisting of two vertices joined by $k$ parallel edges, with one vertex labelled 1 and the other vertex labelled 2. The graph $P_{1}^{\circ k}$ ( $k$-fold concatenation of $P_{1}$ with itself) is equal to $P_{k}$. 
Lemma 3.1. If $H$ has adjacency matrix $A$, then

$$
M\left(P_{k}\right)=A^{k}, \quad M\left(P_{1}^{k}\right)=A^{\circ k},
$$

where $A^{\circ k}$ denotes the $k$-fold Hadamard product of $A$ with itself and $A^{k}$ the $k$-fold ordinary matrix product of $A$ with itself.

Proof. Since $M\left(P_{1}\right)=A, P_{k}=P_{1}^{\circ k}$, by the identities (3.1) we have $M\left(P_{k}\right)=$ $M\left(P_{1}^{\circ k}\right)=M\left(P_{1}\right)^{k}=A^{k}$ and $M\left(P_{1}^{k}\right)=M\left(P_{1}\right)^{\circ k}=A^{\circ k}$.

Lemma 3.1 includes for $k=0$ the fact that, for $P_{0}=K_{1}$ a single vertex with labels 1 and 2 , we have $M\left(K_{1}\right)=I$, where $I$ is the identity matrix (for ordinary matrix multiplication). With $P_{1}^{0}=\bar{K}_{2}$, we also have $M\left(\bar{K}_{2}\right)=J$, where $\bar{K}_{2}$ comprises two isolated vertices, one labelled 1 , the other 2 , and $J$ is the identity matrix for entrywise matrix multiplication (the all-one matrix).

A fundamental lemma (based on the identity (3.1)) for finding contractors for homomorphism functions is the following:

Lemma 3.2. 11] A 2-labelled quantum graph $Z$ is a contractor for hom $(., H)$ if and only if $M(Z)=I$.

Using this lemma we can write down a a quantum path contractor for hom $(\cdot, H)$ when the adjacency matrix of $H$ does not have 0 as an eigenvalue. (Compare 11, Section 4.1].)

Theorem 3.3. Suppose the adjacency matrix $A$ of $H$ has minimal polynomial

$$
p_{A}(t)=p_{0}+p_{1} t+\cdots+p_{\ell} t^{\ell} .
$$

If $p_{0} \neq 0$ (i.e, if 0 is not an eigenvalue of $A$ ), then a contractor for hom $(\cdot, H)$ is given by

$$
Z=-\frac{1}{p_{0}}\left[p_{1} P_{1}+p_{2} P_{2}+\cdots+p_{\ell} P_{\ell}\right]
$$

Proof. If $p_{0} \neq 0$, then $I=-\frac{1}{p_{0}} \sum_{k=1}^{\ell} p_{k} A^{k}$. Hence, using Lemmas 3.1 and 3.2, if $Z=-\frac{1}{p_{0}} \sum_{k=1}^{\ell} p_{k} P_{k}$, then $M(Z)=I$, and $Z$ is a contractor for $\operatorname{hom}(\cdot, H)$.

Similarly, by straightforward polynomial interpolation we have a quantum thick edge contractor for $\operatorname{hom}(\cdot, H)$ when the adjacency matrix of $H$ satisfies a suitable condition:

Theorem 3.4. Suppose the adjacency matrix $A=(\beta(i j))$ of $H$ has the property that

$$
\{\beta(i j): i, j \in V(H), i \neq j\} \cap\{\beta(i i): i \in V(H)\}=\emptyset
$$

and that $|\{\beta(i j): i, j \in V(H)\}|=\ell$.

Then there exist constants $r_{0}, r_{1}, \ldots, r_{\ell}$ (computable by Lagrange interpolation) such that

$$
Z=r_{0} \bar{K}_{2}+r_{1} P_{1}+r_{2} P_{1}^{2}+\cdots+r_{\ell} P_{1}^{\ell}
$$

is a contractor for $\operatorname{hom}(\cdot, H)$.

We are left with the problem of constructing a contractor for hom $(\cdot, H)$ when the adjacency matrix of $H$ has 0 as an eigenvalue or when at least one of its diagonal entries also appears amongst its off-diagonal entries. When $H$ is a weighted Cayley graph we are able to find such a contractor, and the remainder of this section is devoted to showing how.

We begin with the following result of Hoffmann, for which see e.g. [3, Chapter 3]. 
Theorem 3.5. Let $A$ be the adjacency matrix of a graph $H$. The all-one matrix $J$ is a polynomial in $A$ if and only if $H$ is connected and $A$ has eigenvector $\mathbf{1}$ (i.e., $H$ is regular).

Corollary 3.6. Suppose that the adjacency matrix $A$ of a connected graph $H$ on $n$ vertices has eigenvector 1 with eigenvalue $\lambda_{1}$, that its distinct eigenvalues are $\lambda_{1}>\lambda_{2}>\cdots>\lambda_{\ell}$, and that

$$
q(t)=\prod_{2 \leq k \leq \ell}\left(t-\lambda_{k}\right)
$$

Then

$$
J=\frac{n}{q\left(\lambda_{1}\right)} q(A) .
$$

Proof. We have $q(A)=r J$ for some $r \neq 0$ and $q(A)$ has eigenvalues $q\left(\lambda_{1}\right)$ and $q\left(\lambda_{k}\right)=0$ for $2 \leq k \leq \ell$. Since the only nonzero eigenvalue of $r J$ is $r n$, it follows that $r n=q\left(\lambda_{1}\right)$.

The polynomial $q(t)$ in Corollary 3.6 is equal to $p_{A}(t) /\left(t-\lambda_{1}\right)$, where $p_{A}(t)$ is the minimal polynomial of $A$. (Since $A$ is symmetric it is diagonalizable and so each of its Jordan blocks has size 1, which implies that the multiplicity of each root of the minimal polynomial of $A$ is equal to one.)

Suppose now that $A$ has rows and columns indexed by an additive Abelian group $\Gamma$ of order $n$ and has $(i, j)$-entry equal to $(\beta(i-j))$ for all $i, j \in \Gamma$, where $\beta: \Gamma \rightarrow \mathbb{C}$ satisfies $\beta(-i)=\beta(i)$. Thus $A$ is the adjacency matrix of a weighted Cayley graph on $\Gamma$.

Recall the definition of characters and the Fourier transform from Section 2.4 The matrix $A$ has eigenvectors $\left(\chi_{i}(j)\right)_{j \in \Gamma}$ for each $i \in \Gamma$ with corresponding eigenvalue $\widehat{\beta}(i)$. Define $\widehat{A}$ to be the matrix with $(i, j)$-entry the Fourier transform $\widehat{\beta}(i-j)$. For example, $\widehat{I}=J$ and $\widehat{J}=n I$.

Let us call a matrix $\Gamma$-circulant if, like the matrix $A$, it takes the form $(\alpha(i-j))$ for some function $\alpha \in \mathbb{C}^{\Gamma}$. (When $\Gamma$ is cyclic these are circulant matrices as ordinarily defined.)

Lemma 3.7. The map $A \mapsto \widehat{A}$ is an algebra isomorphism of the subalgebra of $\Gamma$-circulant matrices under addition and ordinary matrix multiplication with the subalgebra of $\Gamma$-circulant matrices under addition and entrywise product:

$$
\widehat{A_{1} A_{2}}=\widehat{A}_{1} \circ \widehat{A}_{2}, \widehat{A_{1} \circ A_{2}}=\frac{1}{n} \widehat{A}_{1} \widehat{A}_{2} .
$$

In particular,

$$
\widehat{A^{k}}=\widehat{A}^{\circ k}, \widehat{A^{\circ k}}=\frac{1}{n^{k-1}} \widehat{A}^{k} .
$$

Proof. This is a consequence of the identities (2.3) and (2.4) for the Fourier transform of convolutions and pointwise products. Since the Fourier transform is linear it follows that $A \rightarrow \widehat{A}$ is an algebra isomorphism.

Lemma 3.8. Given $q(A)=q_{0} I+q_{1} A+q_{1} A^{2}+\cdots+q_{\ell-1} A^{\ell-1}$, define

$$
q^{\circ}(A)=q_{0} J+q_{1} A+q_{2} A^{\circ 2}+\cdots+q_{\ell-1} A^{\circ(\ell-1)} .
$$

Then $\widehat{q(A)}=q^{\circ}(\widehat{A})$ and $\widehat{q^{\circ}(A)}=q\left(\frac{1}{n} \widehat{A}\right)$.

Proof. This follows from Lemma 3.7. 
Recall that for $Z \in \mathcal{G}_{2}$ to be a contractor for $\operatorname{hom}(\cdot, H)$ we need $M(Z)=I$. The following lemma is the key to obtaining such a contractor when $H$ has a $\Gamma$-circulant adjacency matrix $A$.

Lemma 3.9. Suppose A has eigenvector 1 with eigenvalue $\lambda_{1}$, minimum polynomial $p_{A}(t)$ and $q(t)=p_{A}(t) /\left(t-\lambda_{1}\right)$. Then

$$
I=\frac{1}{q\left(\lambda_{1}\right)} q^{\circ}(\widehat{A})
$$

Proof. By Corollary 3.6 we have $J=\frac{n}{q\left(\lambda_{1}\right)} q(A)$ and by Lemma 3.8, $\widehat{q(A)}=q^{\circ}(\widehat{A})$. Since $\widehat{J}=n I$, the result follows.

Our main theorem of this section now results:

Theorem 3.10. Suppose that $H$ is a connected graph with adjacency matrix a $\Gamma$ circulant matrix $A$ and that $\widehat{H}$ has adjacency matrix $\widehat{A}$. Suppose further that $A$ has eigenvector 1 with eigenvalue $\lambda_{1}$, has minimal polynomial $p_{A}(t)$, and

$$
\frac{p_{A}(t)}{t-\lambda_{1}}=q(t)=q_{0}+q_{1} t+\cdots+q_{\ell-1} t^{\ell-1} .
$$

Then a contractor for $\operatorname{hom}(\cdot, \widehat{H})$ is given by

$$
Z=\frac{1}{q\left(\lambda_{1}\right)} \sum_{0 \leq k \leq \ell-1} q_{k} P_{1}^{k} .
$$

Proof. By Lemma 3.9 we have $I=\frac{1}{q\left(\lambda_{1}\right)} q^{\circ}(\widehat{A})$. The matrix $\widehat{A}$ is the adjacency matrix of the edge-weighted graph $\widehat{H}$, and $M_{\widehat{H}}(X)$ the $V(\widehat{H}) \times V(\widehat{H})$ matrix whose $(i, j)$-entry is $\operatorname{hom}_{\phi}(X, \widehat{H})$, where $\phi(1)=i, \phi(2)=j$. Since $M_{\widehat{H}}\left(P_{1}^{k}\right)=\widehat{A}^{\circ k}$, the result follows by Lemma 3.2

\section{A Contractor for the Number of $B$-Flows}

In this section we apply Theorem 3.10 to the special case where $H=\operatorname{Cayley}(\Gamma, B)$ for additive Abelian group $\Gamma$, and where $B \subseteq \Gamma \backslash 0$ satisfies $-B=B$.

Let $\delta_{B}$ be the indicator function of $B$. The adjacency matrix $A$ of $H$ has $(i, j)$ entry $\delta_{B}(i-j)$ and eigenvalues $\widehat{\delta}_{B}(i)$ for $i \in \Gamma$. The minimal polynomial $p_{A}(t)$ of $A$ has degree $\ell$ equal to the number of distinct values of $\widehat{\delta_{B}}(c)$ for $c \in \Gamma$. The largest eigenvalue of $A$ is $\widehat{\delta_{B}}(0)=|B|$, belonging to the eigenvector $\mathbf{1}$, and as in Theorem 3.10 we set

$$
\frac{p_{A}(t)}{t-|B|}=q(t)=q_{0}+q_{1} t+\cdots+q_{\ell-1} t^{\ell-1} .
$$

We reach the main theorem of this paper, giving a contractor for $B$-flows.

Theorem 4.1. Let $\Gamma$ be an additive Abelian group of order $n$ and suppose $H=$ Cayley $(\Gamma, B)$ is a connected graph with adjacency matrix $A$.

Let $\widehat{H}$ be the edge-weighted graph on vertex set $\Gamma$ with adjacency matrix $\widehat{A}$.

Then, with the polynomial $q(t)$ defined as in equation (4.1) above,

(i) a contractor for the number of $B$-flows is given by

$$
Z=\frac{n}{q(|B|)} \sum_{0 \leq k \leq \ell-1} q_{k} P_{1}^{k} ;
$$


(ii) a contractor for $n^{c(G)}$ times the number of B-tensions of $G$ is given by

$$
Z=\frac{1}{q_{0}}\left[\frac{q(|B|)}{n} \bar{K}_{2}-\sum_{1 \leq k \leq \ell-1} q_{k} P_{k}\right] .
$$

Proof. Part (i) is a corollary of Theorem 3.10, which gives

$$
\frac{1}{q(|B|)} \sum_{0 \leq k \leq \ell-1} q_{k} P_{1}^{k},
$$

as a contractor for $\operatorname{hom}(\cdot, \widehat{H})$. The number of $B$-flows of $G$ is by Lemma 2.3 equal to $n^{-|V(G)|} \operatorname{hom}(G, \widehat{H})$, and this implies the expression for the contractor as given in the statement of the theorem.

In order to show (ii), by Corollary 3.6 we have

$$
J=\frac{n}{q(|B|)} \sum_{0 \leq k \leq \ell-1} q_{k} A^{k} .
$$

Since $q_{0} \neq 0$, we then obtain

$$
I=\frac{1}{q_{0}}\left[\frac{q(|B|)}{n} J-\sum_{1 \leq k \leq \ell-1} q_{k} A^{k}\right],
$$

and this implies $M_{H}(Z)=I$, where $Z$ is the quantum graph given in the statement of the theorem; i.e., $Z$ is a contractor for $\operatorname{hom}(\cdot, H)$. By Lemma 2.3 the number of $B$-tensions of $G$ is equal to $n^{-c(G)} \operatorname{hom}(G, H)$.

If $B$ does not contain a set of generators for $\Gamma$, then the $\Gamma$-circulant graph $H=\operatorname{Cayley}(\Gamma, B)$ is not connected. The connected components of Cayley $(\Gamma, B)$ correspond to the cosets of the subgroup generated by $B$. Theorem 4.1 is easily extended to this disconnected case as follows:

Theorem 4.2. Let $\Gamma$ be an additive Abelian group of order $n$ and suppose $H=$ Cayley $(\Gamma, B)$ has adjacency matrix $A$ and that $q(t)$ is defined as in equation (4.1) above.

Suppose $H$ has $r$ isomorphic connected components, i.e., its adjacency matrix $A$ is permutation-equivalent to a matrix of the form $I \otimes A_{1}$ for the $r \times r$ identity matrix $I$ and some $n / r \times n / r$ matrix $A_{1}$. Then, a contractor for the number of $B$-flows is given by

$$
Z=\frac{n}{r q(|B|)} \sum_{0 \leq k \leq \ell-1} q_{k} P_{1}^{k} .
$$

Proof. If $B$ generates a subgroup $\Gamma_{1}$ of index $r$ in $\Gamma$, then $\operatorname{Cayley}(\Gamma, B)$ has $r$ components, each isomorphic to a connected Cayley graph $H_{1}=\operatorname{Cayley}\left(\Gamma_{1}, B\right)$ of order $n_{1}=\left|\Gamma_{1}\right|=n / r$. Then $\operatorname{hom}(G, H)=r^{c(G)} \operatorname{hom}\left(G, H_{1}\right)$ is equal to the number of $B$-tensions of $G$ multiplied by $n^{c(G)}$, and $r^{c(G)} n_{1}^{-|V(G)|} \operatorname{hom}\left(G, \widehat{H}_{1}\right)$ is equal to the number of $B$-flows of $G$. If $H_{1}$ has adjacency matrix $A_{1}$, then $H$ has adjacency matrix $I \otimes A_{1}$, where $I$ is the $r \times r$ identity matrix. Let $q_{1}(t)=p_{A_{1}}(t) /(t-|B|)$, where $p_{A_{1}}(t)$ is the minimal polynomial of $A_{1}$. Then $q_{1}(t)=q(t)$, since the eigenvalues of $A_{1}$ coincide with those of $A$ (only the eigenspaces differ). By Corollary 3.6. $J=\frac{n_{1}}{q_{1}(|B|)} q_{1}\left(A_{1}\right)$, whence $I=\frac{n_{1}}{q(|B|)} q^{\circ}\left(\widehat{A_{1}}\right)$ by Corollary 3.9, The result now follows in the same fashion as for the proof of Theorem 4.1 


\section{Conclusion}

In this paper we provide a new and constructive proof for the existence of a contractor for $B$-flows.

Theorem 3.10 provides a general construction of a contractor for a homomorphism function to an edge-weighted Cayley graph. Specifically, it gives a contractor for $\operatorname{hom}(., \widehat{H})$ when $H$ is an edge-weighted Cayley graph of a finite Abelian group $\Gamma$, and $\widehat{H}$ is the edge-weighted Cayley graph with weights given by the Fourier transform of the weights in $H$; Theorem 4.1 applies this to the special case of $B$-flows, where $H=\operatorname{Cayley}(\Gamma, B)$.

We leave for development in another paper [5] the application of this result for choices of Abelian groups and subsets $B$ that are of great interest because of their relevance to long-standing open problems in graph theory. In 9] Jaeger reformulates the Cycle Double Cover Conjecture, Fulkerson's Conjecture, the Petersen Flow Conjecture and related conjectures as $B$-flow problems. Also in the paper [5] we consider the example $B=\{-1,+1\}$ in the additive group $\mathbb{Z}_{n}$ of integers modulo $n$, because the number of $B$-flows here is equal to the number of Eulerian orientations of a (general) graph $G$ when the vertex degrees of $G$ all belong to $\{0,1, \ldots, n-1, n+1\}$. As shown in [10, the number of Eulerian orientations of a graph cannot be expressed in the form $\operatorname{hom}(\cdot, H)$ for a finite edge-weighted graph $H$, and therefore does not have a contractor [11, Example 2.6]. The interesting question arises of whether there is a "limiting contractor" for Eulerian orientations by taking contractors for $\{ \pm 1\}$-flows modulo $n$ as $n \rightarrow \infty$.

Theorem 3.3 gives an algorithm that gives a quantum path contractor for $\operatorname{hom}(\cdot, H)$ when $H$ has a nonsingular adjacency matrix $A$, which involves finding the minimal polynomial of $A$. Theorem 3.4 gives an algorithm that finds a quantum thick edge as a contractor for $\operatorname{hom}(\cdot, H)$ when the adjacency matrix of $H$ has no diagonal entry equal to an off-diagonal entry, which involves using Lagrange interpolation. Left open by these theorems, and only partially covered by Theorems 4.1 and 4.2, is the following:

Problem 5.1. Give an algorithm to find a contractor for $\operatorname{hom}(\cdot, H)$ when $H$ is an edge-weighted connected graph with adjacency matrix $A$ (that has 0 as an eigenvalue and some diagonal entry equal to an off-diagonal entry).

By Theorem 1.4 of [1], in Problem 5.1] it suffices to restrict the search to quantum series-parallel graphs.

Recall that the quantum graph $Z$ is a contractor for the graph parameter $\operatorname{hom}(\cdot, H)$ if $\operatorname{hom}_{\phi}\left(G\left(Z-K_{1}\right), H\right)=0$ for each $G \in \mathcal{G}_{2}$ and $\phi:[2] \rightarrow V(G)$. This equality has (with a switch of homomorphisms from the left and from the right) a similar form to the inequality in the following problem:

Given a $k$-labelled quantum graph $Y$, is it the case that $\operatorname{hom}_{\phi}(Y, G) \geq 0$ for all $k$-labelled graphs $G$ and maps $\phi:[k] \rightarrow V(G)$ ?

Lovász's Seventeenth Problem asks whether such a positive quantum graph $Y$ can be expressed as the sum of squares of labelled quantum graphs (with multiplication being the disjoint union of labelled graphs followed by identification of like-labelled vertices). This has been recently answered in the negative by Hatami and Norine [7]: Problem (5.1) is in general undecidable. It would be interesting to know what the complexity of this problem is when $Y$ is restricted to a subclass of quantum 
graphs, such as series-parallel graphs, and likewise the computational complexity of Problem 5.1 .

Finally, we highlight a particular case of Problem 5.1, since it would be interesting to compare its solution to the three-term deletion-contraction-elimination recursion for the polynomial of Averbouch-Godlin-Makowsky [1].

Problem 5.2. Find a contractor for the graph parameter $\operatorname{hom}\left(G, K_{q-p}^{1}+K_{p}^{y}\right)$, where $K_{p}^{y}$ denotes the complete graph on $p$ vertices with a loop of weight $y$ on each vertex (and similarly for $K_{q-p}^{1}$ ), and the + denotes graph join. (This homomorphism function is equal to $\xi_{G}(q, y-1,(p-q)(y-1))$ in the notation of [1.)

\section{REFERENCES}

[1] I. Averbouch, B. Godlin and J. Makowsky, An extension of the bivariate chromatic polynomial, European J. Combin. 31 (2010), 1-17. MR2552585(2011c:05162)

[2] N.L. Biggs, Interaction Models, London Mathematical Society Lecture Notes Series 30, Cambridge Univ. Press, Cambridge, 1977. MR0674960 (58:32647)

[3] N.L. Biggs. Algebraic Graph Theory. Cambridge Univ. Press, 2nd ed., 1993. MR1271140 (95h:05105)

[4] M. DeVos, J. Nešetřil, and A. Raspaud. On edge-maps whose inverse preserves flows or tensions. In: Graph Theory in Paris (Proceedings of a Conference in Memory of Claude Berge), Trends in Mathematics, pp. 109-138. Birkhäuser, Basel, 2007. MR2279171(2007m:05133)

[5] D. Garijo, A.J. Goodall and J. Nešetřil, On the number of B-flows of a graph, European J. Combin., to appear.

[6] P. de la Harpe and F. Jaeger. Chromatic invariants for finite graphs: theme and polynomial variations. Linear Algebra Appl. 226-228 (1995), 687-722. MR.1344593 (96i:05065)

[7] H. Hatami and S. Norine, Undecidability of linear inequalities in graph homomorphism densities, J. Amer. Math. Soc. 24 (2011), 547-565. MR2748400

[8] W.C. Huffman and V. Pless. Fundamentals of Error-Correcting Codes, Cambridge Univ. Press, Cambridge, 2003. MR 1996953 (2004k:94077)

[9] F. Jaeger, Nowhere-zero flow problems. In: L.W. Beineke and R.J. Wilson, eds., Selected Topics in Graph Theory 3, pp. 71-95. Academic Press, New York, 1988. MR1205397

[10] L. Lovász and B. Szegedy, Limits of dense graph sequences, J. Combin. Theory Ser. B 96:6 (2006), 933-957. MR2274085 (2007m:05132)

[11] L. Lovász and B. Szegedy, Contractors and connectors of graph algebras, J. Graph Theory 60:1 (2009), 11-30. MR2478356 (2010a:05098)

[12] A. Schrijver, Graph invariants in the spin model, J. Combin. Theory Ser. B 99 (2009), 502-511. MR2482968(2010g:05228)

[13] A. Terras, Fourier analysis on finite groups and applications, London Math. Soc. Student Texts 43, Cambridge Univ. Press, Cambridge, 1999. MR1695775 (2000d:11003)

[14] B.L. van der Waerden, Die lange Reichweite der regelmässigen Atomanordnung in Mischkristallen, Z. Physik 118 (1941), 473-488.

[15] J. Wood, Duality for modules over finite rings and applications to coding theory, Amer. J. Math. 121:3 (1999), 555-575. MR1738408(2001d:94033)

Department of Applied Mathematics I, University of Seville, Seville, Spain

E-mail address: dgarijo@us.es

Department of Applied Mathematics (KAM) and Institute of Theoretical Computer

Science (ITI), Charles University, Prague, Czech Republic

E-mail address: goodall.aj@gmail.com

Department of Applied Mathematics (KAM) and Institute of Theoretical Computer Science (ITI), Charles University, Prague, Czech Republid

E-mail address: nesetril@kam.mff.cuni.cz 